OPEN ACCESS

Edited by:

Qingxin Mu,

University of Washington,

United States

Reviewed by:

Zhi-xiang Yuan,

Sichuan University, China

Shiyong Song,

Henan University, China

${ }^{*}$ Correspondence:

Song LI

sol4@pitt.edu

Kechao Zhou

zhoukechao@csu.edu.cn

Specialty section:

This article was submitted to Cancer Molecular Targets

and Therapeutics,

a section of the journal

Frontiers in Pharmacology

Received: 30 April 2018

Accepted: 27 June 2018

Published: 14 August 2018

Citation

Li Z, Sun J, Huang Y, Liu Y, Xu J,

Chen Y, Liang L, Li J, Liao Q, Li S and

Zhou K (2018) A Nanomicellar

Prodrug Carrier Based on Ibuprofen-Conjugated Polymer for Co-delivery of Doxorubicin.

Front. Pharmacol. 9:781

doi: 10.3389/fphar.2018.00781

\section{A Nanomicellar Prodrug Carrier Based on Ibuprofen-Conjugated Polymer for Co-delivery of Doxorubicin}

Zuojun Li1,2, Jingjing Sun ${ }^{3}$, Yixian Huang ${ }^{3}$, Yanhua Liu', Jieni Xü, Yichao Chen ${ }^{3}$, Lei Liang ${ }^{5}$, Jiang $\mathrm{Li}^{3}$, Qiongfeng Liao ${ }^{6}$, Song $\mathrm{Li}^{3 *}$ and Kechao Zhou ${ }^{2 *}$

\footnotetext{
1 Department of Pharmacy, The Third Xiangya Hospital of Central South University, Changsha, China, ${ }^{2}$ State Key Laboratory of Powder Metallurgy, Department of Pharmaceutical Sciences, School of Pharmacy, Central South University, Changsha, China, ${ }^{3}$ Center for Pharmacogenetics, Department of Pharmaceutical Sciences, School of Pharmacy, University of Pittsburgh, Pittsburgh, PA, United States, ${ }^{4}$ Department of Pharmaceutics, School of Pharmacy, Ningxia Medical University, Yinchuan, China, ${ }^{5}$ Guangdong Second Provincial General Hospital, Guangzhou, China, ${ }^{6}$ School of Pharmaceutical Sciences, Guangzhou University of Chinese Medicine, Guangzhou, China
}

Ibuprofen (IBU) is a non-steroidal anti-inflammatory drug (NSAID), which is widely used to reduce fever and treat inflammation and acute pain. Recently, its application in cancer treatment is also being explored. In this work, we synthesized a well-defined IBU-based amphiphilic diblock copolymer via reversible addition fragmentation transfer (RAFT) polymerization of IBU-based vinyl monomer. The amphiphilic copolymer POEG$b$-PVBIBU (denoted as POVI) was composed of a hydrophilic poly(oligo(ethylene glycol)) block and a hydrophobic IBU-bearing prodrug block, which was able to self-assemble into prodrug nanomicelles. In addition, it could serve as a carrier to co-load other drugs including doxorubicin (DOX), paclitaxel (PTX), and docetaxel (DTX). By using DOX as a model anti-cancer drug, the delivery function of POVI carrier, including the drug release, in vitro cytotoxicity, cellular uptake, and in vivo antitumor activity, was evaluated. DOXloaded POVI micelles exhibited sustained release of DOX. Besides, DOX/POVI micelles were effectively taken up by tumor cells with an efficiency comparable to that of free DOX. Moreover, in vivo studies showed that POVI carrier itself had modest antitumor activity. After loading DOX, the antitumor activity was significantly increased, which was significantly higher than that of free DOX. Our results suggest that POVI polymer represents a simple and effective dual-functional carrier for co-delivery of IBU and DOX to improve the anticancer activity.

Keywords: ibuprofen, nanomicellar, prodrug, carrier, doxorubicin, co-delivery 


\section{INTRODUCTION}

The clinical applications of many antitumor drugs, such as doxorubicin (DOX), paclitaxel (PTX), and docetaxel (DTX), were limited by their low water solubility, poor bioavailability, and high toxic side effects (Lee et al., 2011; Senevirathne et al., 2016). To overcome these problems, various nanocarriers including micelles, dendrimers, and liposomes have been designed and developed for delivery of these hydrophobic antitumor drugs (Cho et al., 2008; Shen et al., 2010; Wei et al., 2013; Koudelka et al., 2015; Wang et al., 2015, 2017; Miao et al., 2017). Due to the small size, good stability, and unique core-shell structure, polymeric micelles have gained tremendous interest in the drug delivery field (Gaucher et al., 2005; Rösler et al., 2012). The hydrophobic core of micelles can encapsulate hydrophobic antitumor drugs via hydrophobic interactions, and the hydrophilic shell endows the micelles colloidal stability and protects the loaded drug from premature burst release.

It is well known that the constructing material, especially the hydrophobic domain of the polymeric micelles, plays a very important role in the delivery function of carriers (Zhang et al., 2014). Recently, increasing attention has been focused on the usage of bioactive compounds as hydrophobic moieties of polymeric carriers. These carriers themselves possess biological activity, which are termed as prodrug carriers (Craparo et al., 2013; Chen et al., 2016; Sun et al., 2017a). More importantly, some bioactive compounds with special structures in the prodrug carriers could interact with the co-loaded drug via $\pi-\pi$ stacking effect, thereby improving the drug loading capacity (DLC; Sun et al., 2017b).

Non-steroidal anti-inflammatory drugs (NSAIDs), such as ibuprofen (IBU), indomethacin, and aspirin, are widely used to treat fever, arthritis, and rheumatic diseases. The antiinflammatory effect of NSAIDs comes from the inhibition of the cyclooxygenase (COX) enzymes, including COX-1 and COX-2, which leads to the decrease of the production of prostaglandin, an important signaling molecule in the inflammation (Lim et al., 1999). Recently, accumulating evidences demonstrate that NSAIDs not only have anti-inflammatory effect, but also hold great potential in the prevention and treatment of several types of cancers (Ulrich et al., 2006). It has been suggested that the antitumor activity of NSAIDs can be partially explained by COX inhibition of prostaglandin synthesis (Ahnen, 1998). Other COX-independent mechanisms including inhibition of cell cycle progression (Gately and Kerbel, 2003) and induction of apoptosis (Davies et al., 2002) also contribute to the antitumor activity of NSAIDs. IBU is a commonly used NSAID that has been proven to inhibit proliferation of many tumor cells (Dial et al., 2006; Bonelli et al., 2011). Studies also demonstrate that IBU exhibited superior antitumor effect compared to other NSAIDs, mainly through the alteration of cell-cycle and induction of apoptosis (Andrews et al., 2002; Janssen et al., 2008). Moreover, compared to conventional anticancer drugs, IBU shows decreased side effect. Due to the potential of IBU in cancer treatment, IBUconjugated prodrug polymers with various molecular structures have been developed to improve the water solubility and bioavailability of IBU. For example, Hasegawa and colleagues synthesized a series of amphiphilic diblock copolymers (PEGPIBU) containing a hydrophilic poly(ethylene glycol) block and a hydrophobic IBU-bearing prodrug block (Hasegawa et al., 2013). By adjusting the length of hydrophobic IBU block, these polymers could self-assemble to form micelles with different morphologies. Wang et al. (2014) prepared another amphiphilic micellar carrier based on IBU-conjugated polymer for delivery of IBU. They found that in addition to the chemical conjugation, IBU could also be physically encapsulated into the micelles.

Although NSAIDs have potential antitumor activity, very high dosages of NSAIDs are needed to achieve a modest antitumor effect (Smalley and DuBois, 1997). Combination treatment of NSAIDs with other chemotherapeutic drugs is still necessary for cancer therapy (Endo et al., 2014; Lee et al., 2016). Recently, our group prepared a nanomicellar carrier that was self-assembled from PEG-Fmoc-IBU conjugate for co-delivery of IBU and PTX (Zhao et al., 2016). The IBU-based carrier showed synergistic antitumor effect with PTX, but there is only one IBU unit per carrier molecule, and thereby, large amounts of carriers were needed to deliver enough IBU dosage to tumor tissues, which might cause the unfavorable side effects.

Thus, in this work, we developed another IBU-containing polymeric carrier system with increased number of IBU units per polymer molecule via a simple synthesis method. We synthesized a POEG-b-PVBIbu diblock copolymer with a hydrophilic POEG block and a hydrophobic IBU block by reversible addition fragmentation transfer (RAFT) polymerization. The IBU-based prodrug polymer can form stable micelles with multiple IBU hydrophobic moieties in the core, which increases the solubility of IBU and allows more IBU being delivered into the tumor. Additionally, the micelle can serve as a carrier to encapsulate other hydrophobic chemotherapeutic drugs including DOX, DTX, and PTX. The size distribution and morphologies of drug-loaded micelles were evaluated. By using DOX as a model drug, the drug release, cellular uptake and antitumor activity were investigated in vitro and in vivo.

\section{MATERIALS AND METHODS}

\section{Materials}

Vinylbenzyl chloride, 2, 2-Azobis(isobutyronitrile) (AIBN), 1,4-dioxane, trypsin-EDTA solution, 3-(4,5-dimethylthiazol-2yl)-2,5-diphenyl tetrazolium bromide (MTT), and Dulbecco's Modified Eagle's Medium (DMEM) were all purchased from Sigma-Aldrich (St. Louis, MO, United States). AIBN was purified by recrystallization in anhydrous ethanol. DOX. $\mathrm{HCl}$ and DTX were purchased from LC Laboratories (Woburn, MA, United States). PTX was purchased from AK Scientific Inc. (Union City, CA, United States). Fetal bovine serum (FBS) and penicillin-streptomycin solution were all purchased from Invitrogen (NY, United States). POEG macroCTA were prepared as previously reported (Sun et al., 2016a). 


\section{Synthesis of Ibu-Monomer}

Vinylbenzyl chloride ( 1 eq.), IBU (1.5 eq.), and $\mathrm{K}_{2} \mathrm{CO}_{3}$ (2 eq.) were dissolved in DMF (5 eq.). The mixture was stirred at $50^{\circ} \mathrm{C}$ for $24 \mathrm{~h}$, and then equal volume of water was added. The crude product was extracted by DCM for three times, and then purified by silica gel column chromatography.

\section{Synthesis of POEG-b-PVBIbu Polymers}

Ibu-monomer (180 mg, $0.559 \mathrm{mmol})$, POEG macroCTA (280 mg, $0.0372 \mathrm{mmol}$ ), AIBN (2 mg, $0.0124 \mathrm{mmol}$ ), and $2 \mathrm{~mL}$ dried 1, 4-dioxane were placed in a Schlenk tube. The mixture was degassed under $\mathrm{N}_{2}$ by three freeze-pumpthaw cycles, and then immersed into an oil bath at $90^{\circ} \mathrm{C}$. After $24 \mathrm{~h}$, the polymerization was quenched by placing the Schlenk tube into liquid nitrogen. The polymer mixture was precipitated in petroleum ether for three times and dried in vacuum.

\section{Characterization of the Synthesized Monomer and Polymers}

${ }^{1} \mathrm{H}$ NMR spectrum was conducted on a Varian 400 FT-NMR spectrometer at $400.0 \mathrm{MHz}$ with $\mathrm{CDCl}_{3}$ as the solvent. Molecular weight $\left(M_{\mathrm{n}}\right.$ and $\left.M_{\mathrm{w}}\right)$ and polydispersity index $\left(M_{\mathrm{w}} / M_{\mathrm{n}}\right)$ of the synthesized polymers were determined by gel permeation chromatography (GPC) equipped with a Waters 2414 refractive index detector, a Waters 515 HPLC pump, and a Waters 717 Plus Autosampler. THF was used as the eluent with a flowing rate of $1.0 \mathrm{~mL} / \mathrm{min}$ at $35^{\circ} \mathrm{C}$. A series of polystyrene standards with narrow molecular weight distribution were used for calibration.

\section{Preparation of Drug-Free Micelle and Drug-Loaded Micelles}

The blank POVI micelles and drug-loaded micelles were prepared by dialysis method. The polymer POVI and drug (DOX, PTX, or DTX) with certain mass ratio were dissolved in DMSO, which were then transferred to dialysis bags (MWCO $3.5 \mathrm{kDa}$ ), and dialyzed against PBS for $24 \mathrm{~h}$. The size distributions and morphologies of blank and drug-loaded micelles were measured by dynamic light scattering (DLS) and transmission election microscopy with negative staining.

Doxorubicin concentration was detected by Fluorescence Microplate Reader with excitation wavelength of $490 \mathrm{~nm}$ and emission wavelength of $590 \mathrm{~nm}$. PTX concentration was measured by reverse phase high-performance liquid chromatography (RP-HPLC) with a mobile phase of methanol/water $(70: 30 \mathrm{v} / \mathrm{v})$ at the flow rate of $1.0 \mathrm{~mL} / \mathrm{min}$, and UV detection at $227 \mathrm{~nm}$. DTX concentration was detected by RP-HPLC with a mobile phase of acetonitrile/water (50:50 $\mathrm{v} / \mathrm{v}$ ) at the flow rate of $1.0 \mathrm{~mL} / \mathrm{min}$, and UV detection at $230 \mathrm{~nm}$.

Drug loading capacity and drug loading efficiency (DLE) were calculated according to the following formula:

DLC $(\%)=$ [weight of drug loaded $/($ weight of polymer + drug used) $] \times 100 \%$

DLE $(\%)=$ (weight of loaded drug/weight of input drug) $\times 100 \%$.

\section{Critical Micelle Concentration (CMC) of POVI Micelle}

Critical micelle concentration (CMC) of POVI micelle was measured using Nile red as a fluorescence probe

(i)

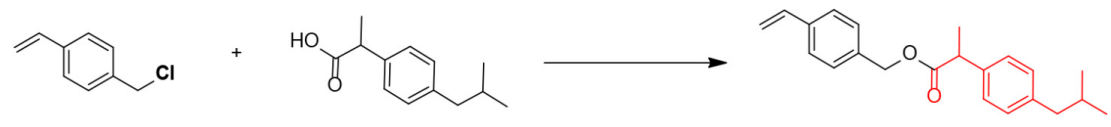

(ii)
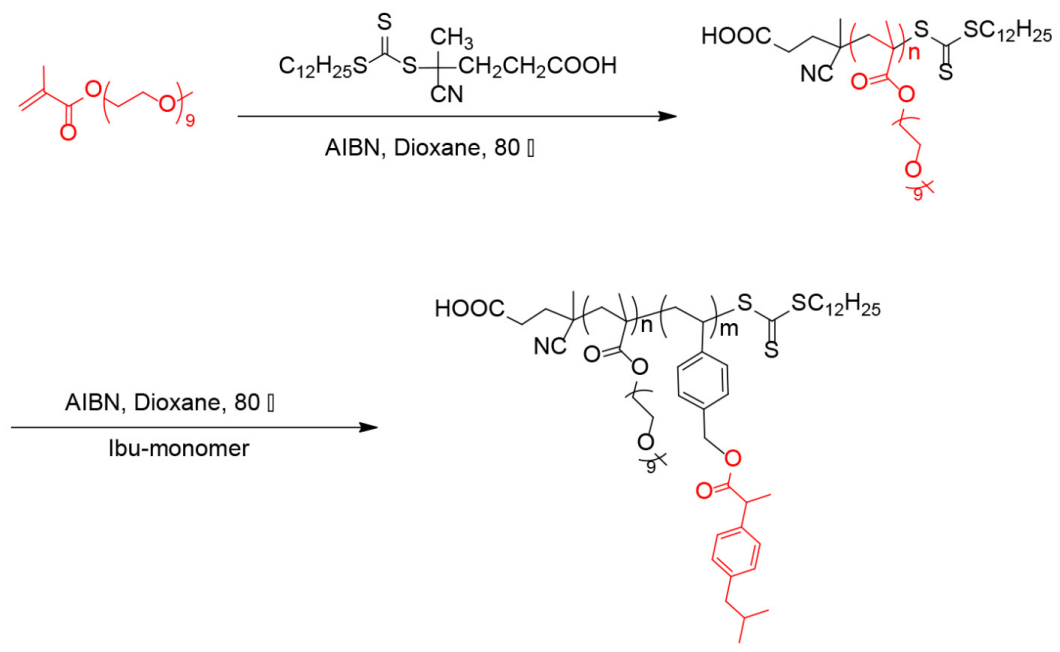

SCHEME 1 | Synthesis of the IBU-monomer and POEG-b-PVBIBU (POVI) polymers via RAFT polymerization. 


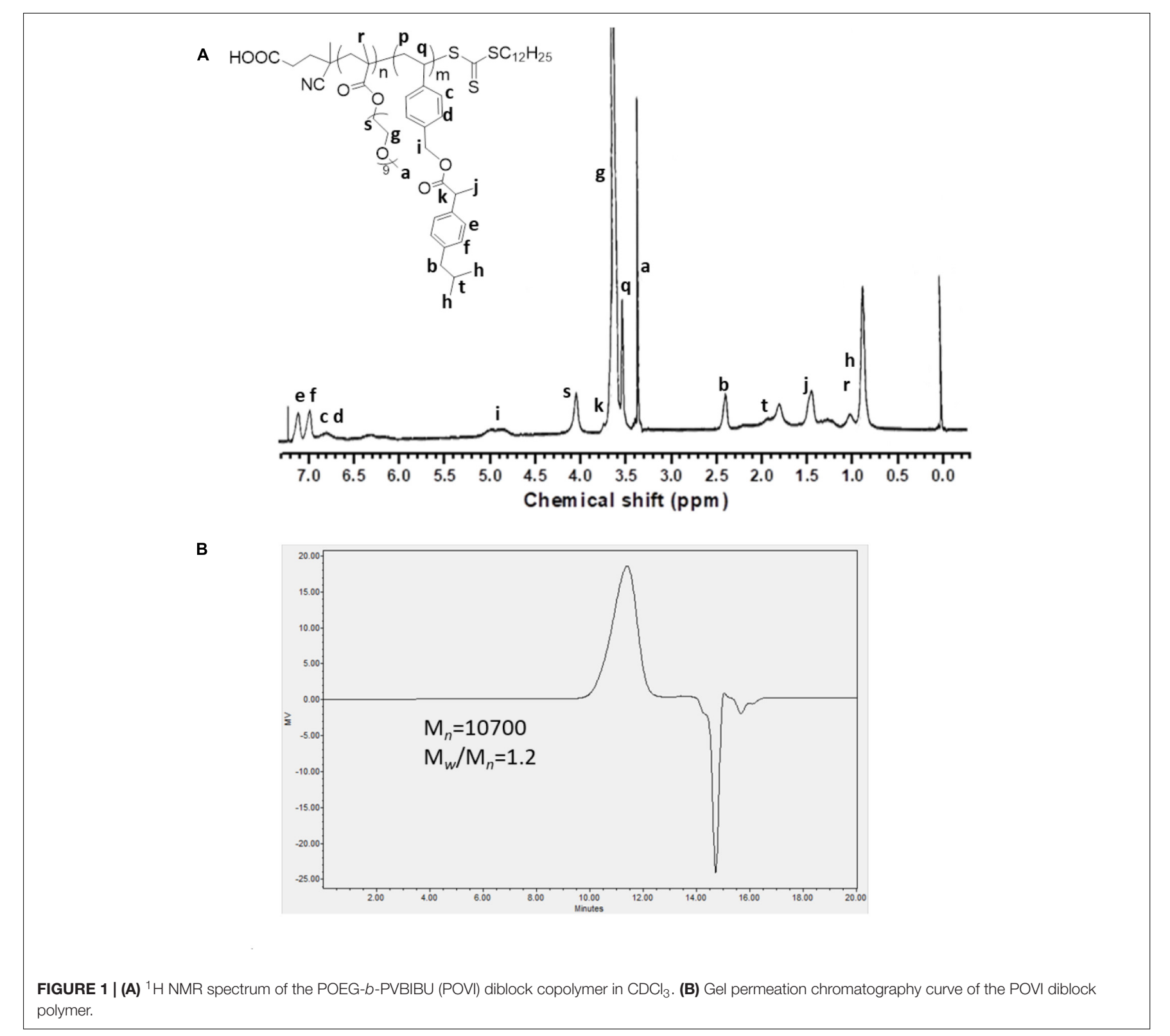

TABLE 1 | Physicochemical characterization of POVI blank micelle and drug-loaded micelles.

\begin{tabular}{lccccc}
\hline Micelles & Size $(\mathbf{n m})^{\mathbf{b}}$ & PDI $^{\mathbf{c}}$ & $\begin{array}{c}\text { Carrier: DOX } \\
\text { mass ratio }\end{array}$ & DLE\%d & DLC\% $^{\mathbf{d}}$ \\
\hline POVI $^{\mathrm{a}}$ & 82.35 & 0.207 & - & - & - \\
DOX/POVI & 92.78 & 0.194 & $10: 1$ & $69.98 \%$ & $6.4 \%$ \\
PTX/POVI & 106.7 & 0.281 & $20: 1$ & $72.90 \%$ & $3.5 \%$ \\
DTX/POVI & 98.30 & 0.204 & $20: 1$ & $41.74 \%$ & $2.0 \%$ \\
\hline
\end{tabular}

${ }^{a}$ Concentration of blank micelle was $20 \mathrm{mg} / \mathrm{mL}$. ${ }^{b}$ Measured by DLS. ${ }^{c} P D I$, polydispersity index; ${ }^{d} D L C \%$, drug loading capacity; DLE\%, drug loading efficiency.

(Klaikherd et al., 2009; Sun et al., 2016b); $10 \mu \mathrm{L}$ Nile red in chloroform was added into each tube, and the solvent was removed by air flow and vacuum pump. Polymer solution
$(120 \mu \mathrm{L})$ with concentrations ranging from $1 \times 10^{-4}$ to $0.5 \mathrm{mg} / \mathrm{mL}$ was added to each tube, and incubated overnight. The fluorescence intensity of each sample was detected by a Synergy H1 Hybrid Multi-Mode Microplate Reader (Winooski, VT, United States) at a wavelength of 480/620 nm (excitation/emission).

\section{In vitro Drug Release Study}

Doxorubicin-loaded POVI micelles $(0.5 \mathrm{mg} \mathrm{DOX} / \mathrm{mL})$ in PBS $(\mathrm{pH}=7.4)$ was placed in a dialysis bag $(\mathrm{MWCO}=12 \mathrm{kDa}$, Spectrum Laboratories), and incubated in a $200-\mathrm{mL}$ beaker with PBS containing $0.5 \%(\mathrm{w} / \mathrm{v})$ Tween 80 , with gentle shaking $(100 \mathrm{rpm} / \mathrm{min})$ at $37^{\circ} \mathrm{C}$. DOX solution in saline with the same concentration was used as a control. The concentration of DOX outside the dialysis bag was measured 
by a fluorescence microplate reader at designated time points and the values were reported as the means of triplicate samples.

\section{Cell Culture}

Mouse metastatic breast cancer cell line 4T1.2, human breast cancer cell line MCF-7, and androgen-independent human prostate cancer cell line $\mathrm{PC}-3$ were cultured at $37^{\circ} \mathrm{C}$ in DMEM containing $10 \%$ FBS and $1 \%$ penicillin-streptomycin in a humidified environment with $5 \% \mathrm{CO}_{2}$.

\section{In vitro Cytotoxicity Study}

4T1.2 (1500 cells/well), MCF-7 (4000 cells/well), or PC-3 (2500 cells/well) were seeded in 96-well plates and incubated for $24 \mathrm{~h}$. Then the cells were treated with various concentrations of drugfree POVI micelles, DOX-loaded POVI micelles, or DOX. After incubation for $72 \mathrm{~h}, 20 \mu \mathrm{L}$ of MTT in PBS $(5 \mathrm{mg} / \mathrm{mL})$ was added into each well and further incubated for $4 \mathrm{~h}$. The medium was then removed, and DMSO was added to solubilize the MTT formazan. The absorbance of each well was measured with a microplate reader at a wavelength of $550 \mathrm{~nm}$ and a reference wavelength of $630 \mathrm{~nm}$. Untreated cells were used as a control. Cell viability was calculated as $\left[\left(\mathrm{OD}_{\text {treat }}-\mathrm{OD}_{\text {blank }}\right) /\left(\mathrm{OD}_{\text {control }}\right.\right.$ $\left.\left.-\mathrm{OD}_{\text {blank }}\right) \times 100 \%\right]$.

\section{Intracellular Trafficking}

4 T1.2 cells $(15,000 /$ well) were seeded in glass bottom dishes (in vitro Scientific, United States), and incubated overnight. The cells were treated with free DOX and DOX/POVI micelles (DOX concentration: $15.5 \mu \mathrm{g} / \mathrm{mL}$ ) for 2 and $4 \mathrm{~h}$ separately. Then cells were stained with Hoechst 3342 for 15 min, and washed with cool PBS for three times. The intracellular distributions of different DOX formulations were observed under a confocal laser scanning microscope (CLSM, FluoView 1000, Olympus, Japan).

\section{Animals}

Female BALB/c mice (6-8 weeks) were purchased from Charles River (Davis, CA, United States). All animals were housed under pathogen-free conditions according to Association for Assessment and Accreditation of Laboratory Animal Care (AAALAC) guidelines. All animal-related experiments were performed in full compliance with institutional guidelines and approved by the Animal Use and Care Administrative Advisory Committee at the University of Pittsburgh.

\section{In vivo Therapeutic Study}

A syngeneic murine breast cancer model (4T1.2) was used to evaluate the therapeutic efficacy of DOX-loaded POVI micelles. $4 \mathrm{~T} 1.2$ cells $\left(2 \times 10^{5}\right.$ cells/mouse $)$ were inoculated s.c. at the right flank of female $\mathrm{BALB} / \mathrm{c}$ mice. When the tumor volume reached $\sim 50 \mathrm{~mm}^{3}$ (day 0 ), mice were randomly divided into four groups $(n=3)$ and received i.v. administration of saline (control), POVI micelles, free DOX, and DOX-loaded POVI micelles, respectively, on days $0,3,6,9,12,15$, and 18 . The DOX dosage for free DOX and DOX-loaded POVI micelles was $5 \mathrm{mg} \mathrm{DOX} / \mathrm{kg}$. The dosage for POVI micelles was $73 \mathrm{mg}$ POVI $/ \mathrm{kg}$, which was the same as that of POVI in DOXloaded POVI micelles. Tumor volumes were measured with digital caliper and calculated as $V=\left(L \times W^{2}\right) / 2$, where $L$ is the longest and $W$ is the shortest tumor diameters $(\mathrm{mm})$. Each group was compared by relative tumor volume (RTV $=V / V_{0}, V_{0}$ was the tumor volume prior to first treatment). Mice were sacrificed when the tumor volume reached $\sim 2000 \mathrm{~mm}^{3}$. The size and weight of tumor stripped from the

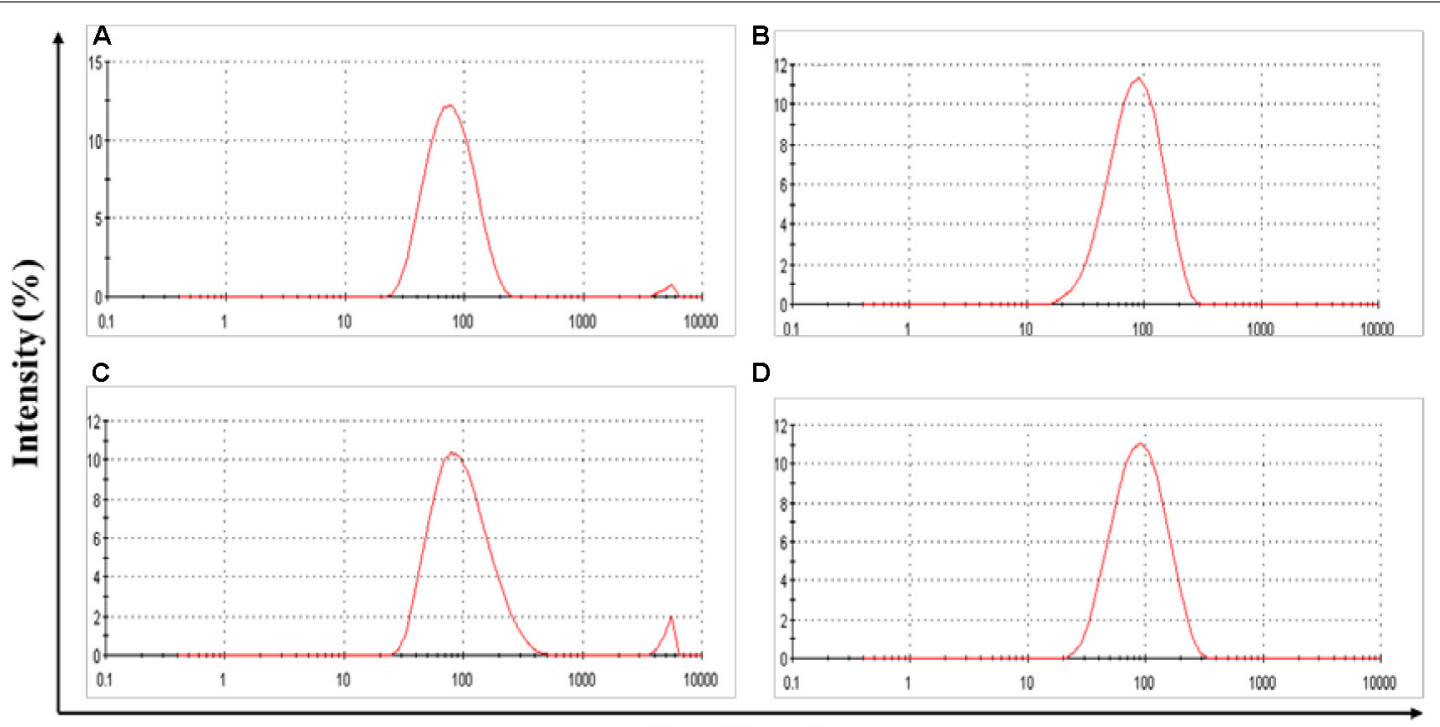

Size (d. nm)

FIGURE 2 | Size distribution of POVI (A), DOX/POVI (B) (mass ratio of micelle to DOX = 10:1), PTX/POVI (C), and DTX/POVI micelles (D) (mass ratio of micelle to PTX/DTX $=20: 1)$. 
mice were measured. To evaluate the potential toxicity, the body weights were also monitored during the entire course of treatment.

\section{Histochemical Staining}

After in vivo therapeutic study, tumor tissues were excised and preserved in $4 \%$ formaldehyde in PBS, followed by embedment in paraffin. The paraffin-embedded tumor samples were cut into thin slices of $5 \mu \mathrm{m}$ with an HM 325 Rotary Microtome. Then the slices were stained with hematoxylin and eosin (H\&E) for histopathological examination under a Zeiss Axiostar plus Microscope (PA, United States).

\section{Statistical Analysis}

All results were reported as the mean \pm SD unless otherwise indicated. Statistical analysis was performed with Student's $t$-test for two groups, and one-way ANOVA for multiple groups, followed by Newman-Keuls test if $P<0.05$. In all statistical analysis, $P<0.05$ was considered statistically significant.

\section{RESULTS}

\section{Synthesis of POEG-b-PVBlbu Polymers}

Reversible addition fragmentation transfer polymerization of functional monomer has become an attractive strategy to obtain well-defined functional polymers for drug/gene delivery (Sun et al., 2013a,b; Tucker et al., 2015). In this work, we synthesized a well-defined IBU-based prodrug polymer via RAFT polymerization of IBU-conjugated monomer, and investigated its function as a dual-functional carrier for co-delivery of other chemotherapeutic drugs.

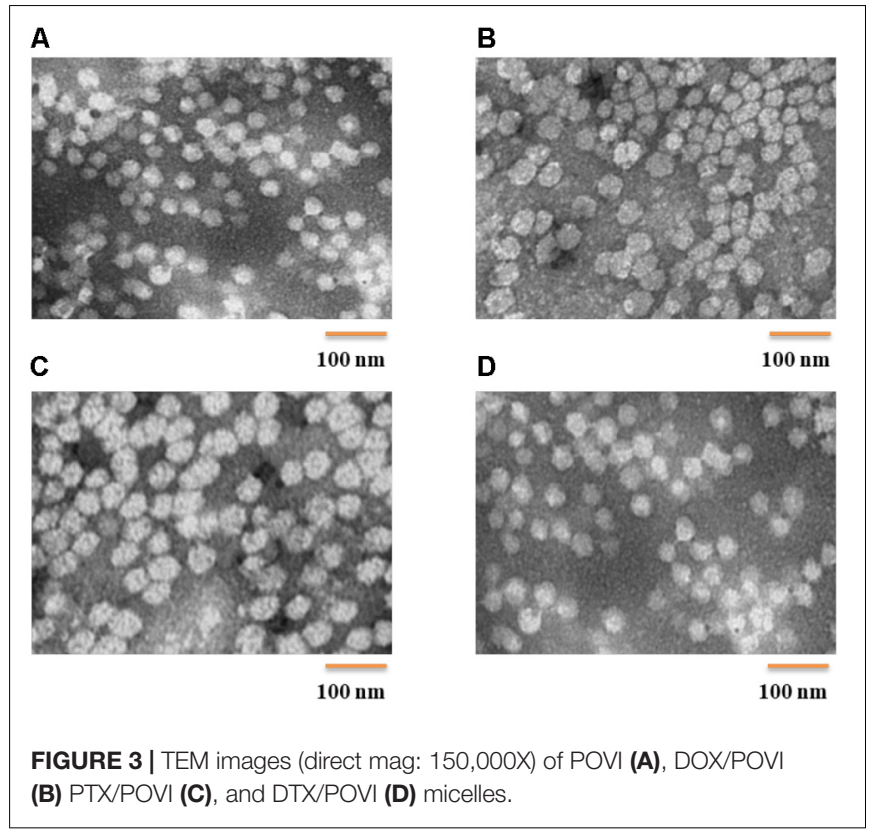

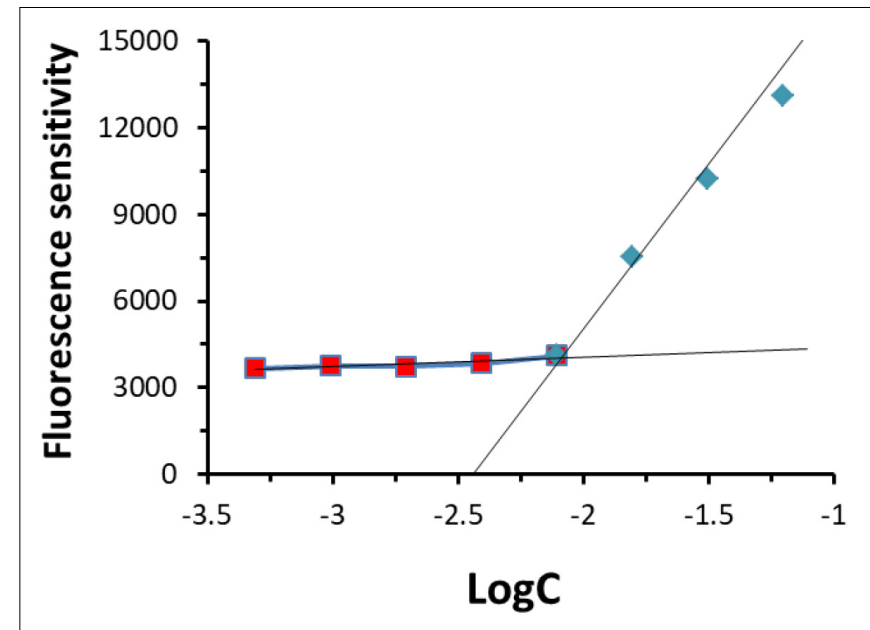

FIGURE 4 | Critical micelle concentration (CMC) of POVI micelle.

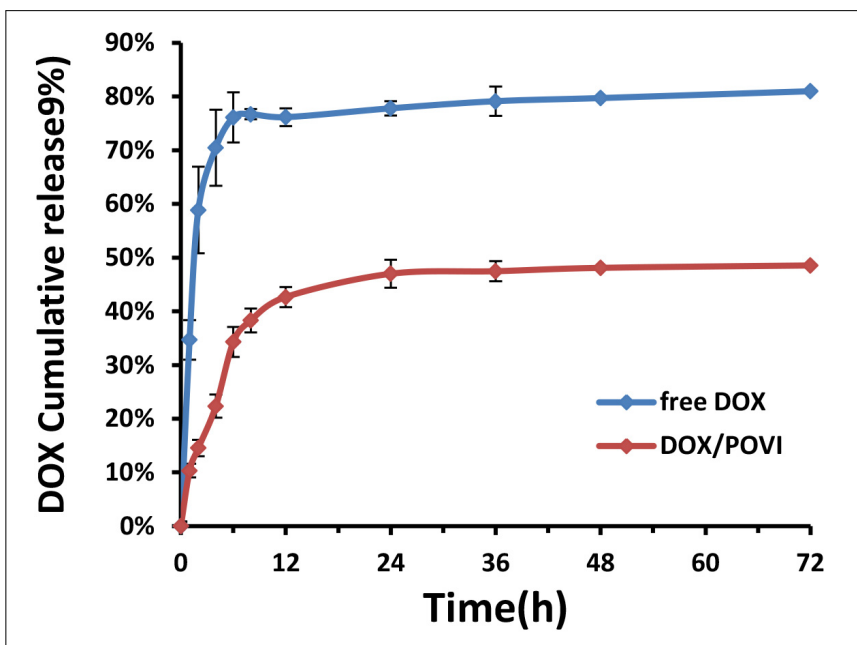

FIGURE 5 | DOX release from DOX-loaded POVI micelles via a dialysis method. PBS solution was used as release medium, and DOX concentrations were analyzed at 0, 1, 2, 4, 6, 8, 12, 24, 36, 48, and $72 \mathrm{~h}$.

As shown in Scheme 1, we first designed and synthesized a vinylbenzyl derivative of IBU (IBU-monomer) where IBU was conjugated with vinylbenzyl chloride via a hydrolyzable ester linkage. Then, the macro-chain transfer agent POEG macroCTA was synthesized by RAFT polymerization as previously reported (Sun et al., 2016a), which further initiated the polymerization of IBU-monomer to give the POEG- $b$-PVBIbu block copolymers. The structures of the monomer and polymer were confirmed by ${ }^{1} \mathrm{H}$ NMR (Figure 1). As shown in Figure $\mathbf{1 A}$, the average degree of polymerization (DP) of the Ibu-monomer was determined to be 12 by comparing the intensities of $I_{\mathrm{b}}$ and $I_{\mathrm{a}}$. GPC showed unimodal peaks for the polymer with number-average molecular weight $M_{\mathrm{n}}$ of 10,700, and polydispersity of 1.20 (Figure 1B), which indicated the successful synthesis of the well-defined POEG- $b$-PVBIbu (POVI) block copolymers. 


\section{Preparation and Physicochemical Characterization of POVF Blank Micelles and Drug-Loaded Micelles}

POVF blank micelles and drug-loaded micelles (including DOX/POVI, PTX/POVI, and DTX/POVI) were successfully prepared by dialysis method, and the physicochemical characterization is summarized in Table 1. DLS analysis showed that POVF polymers could form nano-sized micelles with a diameter of $82 \mathrm{~nm}$. The sizes of micelles slightly increased to 92, 106, and $98 \mathrm{~nm}$ after loading of DOX, PTX, and DTX, respectively (Figure 2), which may be due to that the encapsulated hydrophobic drugs enlarged hydrophobic core of the micelles. The morphologies of the blank and drug-loaded micelles were evaluated by transmission electron microscopy (TEM). As shown in Figure 3, these micelles are spherical in morphology with uniform size. DLE and capacity were measured by a fluorescence microplate reader. As shown in the Table 1, POVI polymer was able to load DOX with a loading capacity of $6.4 \%$. In addition, POVI polymers could also serve as a carrier to load other drugs such as PTX and DTX.

The CMC value of POVF micelles was evaluated with Nile red as a fluorescence probe. As shown in Figure 4, the CMC of POVI micelles was measured to be $9.8 \mathrm{mg} / \mathrm{L}$. The low CMC suggests a good stability of our micelles following dilution in the blood after i.v. administration.

\section{Release Kinetics of DOX}

The release kinetics of DOX from DOX/POVI micelles was evaluated using a dialysis method, and free DOX solution was used as a control. As shown in Figure 5, free DOX solution showed a burst DOX release of almost $80 \%$ within the first $12 \mathrm{~h}$, while DOX/POVI micelles showed a slow DOX release of about $43 \%$. Even after $72 \mathrm{~h}$, less than $50 \%$ of DOX was released from DOX/POVI micelles.

\section{In vitro Cytotoxicity of DOX/POVI}

In vitro cytotoxicity of DOX/POVI micelles was evaluated in 4T1.2, MCF7, and PC3 cell lines. As shown in Figure 6, POVI carrier did not show any remarkable cytotoxicity among all cell lines tested. DOX/POVI micelles and DOX solution exhibited potent cytotoxicity in a concentrationdependent manner. DOX/POVI micelles exhibited lower cytotoxicity than free DOX in the three cell lines, which is likely due to the slow release of DOX from DOX/POVI micelles.

\section{Intracellular Trafficking}

Intracellular trafficking and distribution of DOX/POVF micelles were studied by confocal laser scanning microcopy (CLSM). 4T1.2 cells were incubated with free DOX and DOX/POVF micelles for 2 and $4 \mathrm{~h}$, respectively. At $2 \mathrm{~h}$,

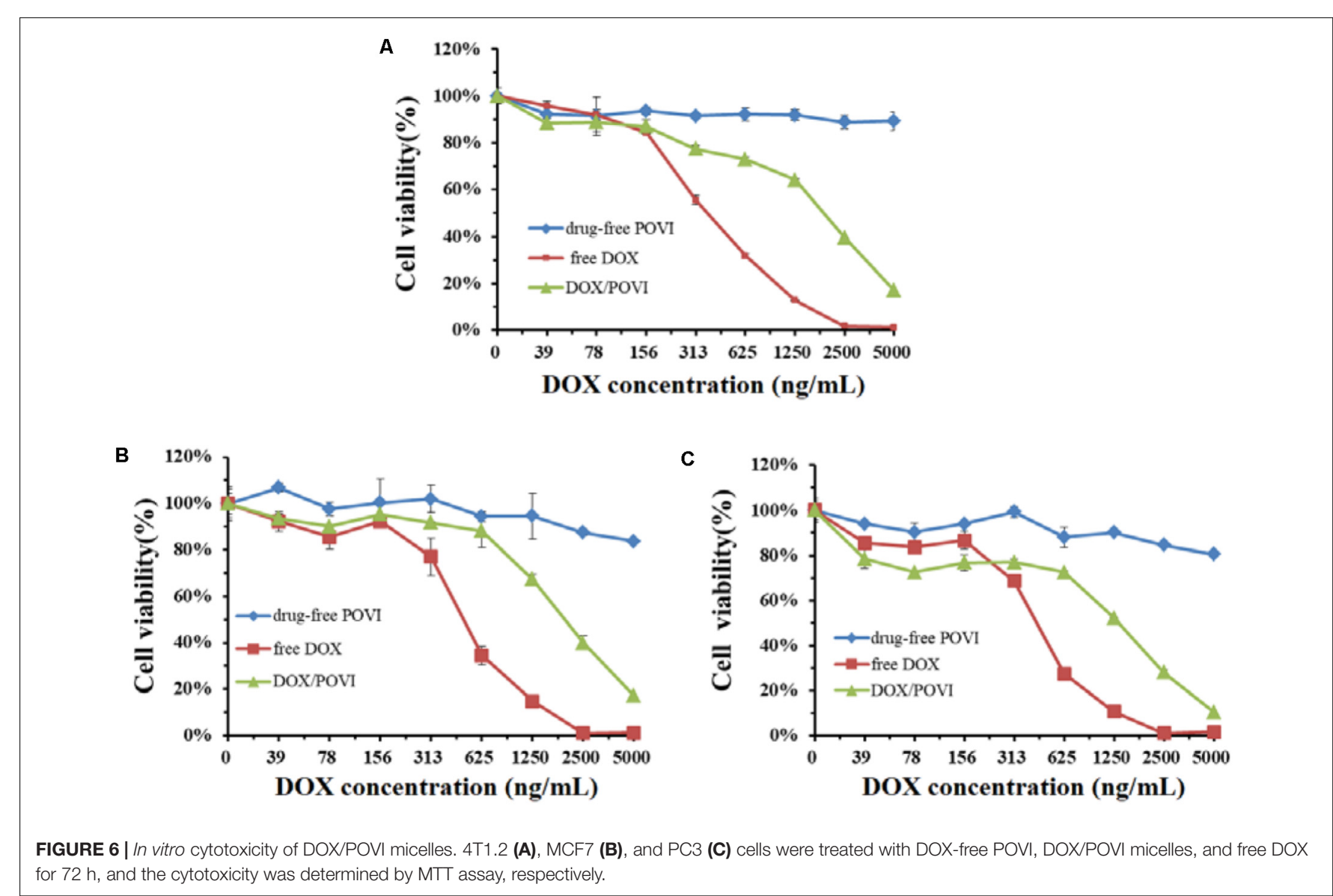




Dox

cells treated with DOX/POVF micelles exhibited strong DOX fluorescence signals around the nucleus, similar to the cells treated with free DOX (Figure 7A). At $4 \mathrm{~h}$, increased signals of red fluorescence appeared in nuclei, both for DOX/POVF micelle group and free DOX group (Figure 7B).

\section{In vivo Therapeutic Efficacy Evaluation}

The in vivo therapeutic efficacy of DOX/POVI micelles was evaluated in the $4 \mathrm{~T} 1.2$ breast tumor-bearing $\mathrm{BALB} / \mathrm{c}$ mice. The mice were treated with saline, POVI carrier, free DOX, and DOX/POVI micelles by i.v. injection, respectively. As shown in Figure 8A, DOX exhibited a modest tumor growth inhibition at a dose of $5 \mathrm{mg}$ DOX $/ \mathrm{kg}$. DOX/POVI micelles were more effective than DOX in inhibiting tumor growth $(p<0.05)$ at the same dose. Interestingly, POVI carrier alone also showed tumor growth inhibition effect, although it was less effective than DOX.

No significant changes were found in the body weights of mice following various treatments (Figure $\mathbf{8 B}$ ), indicating that all treatments were well tolerated. Figure $\mathbf{8 C}$ shows the photographs of tumors removed from the mice after various treatments. Compared to saline treatment group, DOX/POVI treatment led to an obvious decrease in tumor size. The tumor growth inhibition rate of DOX/POVI micelles is calculated to be significantly higher than those of free DOX and POVI blank micelles $(P<0.05$; Figure 8D).

Figure 9 shows the images of H\&E-stained slices of tumors collected after the completion of in vivo therapy study. Tumors treated with saline showed normal tumor morphology with large nuclei. In contrast, tumors in other treatment groups showed shrunk nuclei. Among them, DOX/POVF treatment resulted in the most significant tumor necrosis.

\section{DISCUSSION}

Ibuprofen is one type of NSAID that not only possesses antiinflammatory effect, but also demonstrates great potential in inhibiting proliferation of various types of tumor cells (Dial et al., 2006; Bonelli et al., 2011). Currently, combination of IBU with chemotherapeutic drugs is being evaluated as a new regimen for clinical cancer treatment. However, free drugs are readily eliminated in the blood and they lack tumor targeting. Thus, co-delivery of IBU and a chemotherapeutic drug into tumors using a delivery vehicle is highly needed. Previously, we reported a nanomicellar carrier based on PEGFmoc-IBU conjugate for co-delivery of IBU and PTX (Zhao et al., 2016). Although PEG-Fmoc-IBU carrier was effective in loading and delivering PTX into tumors, the efficiency of delivery of IBU itself was relatively low because there was only one IBU unit per polymer molecule. In the present work, we developed a new IBU-conjugated polymeric carrier POVI with increased units of IBU per carrier molecule via a facile RAFT polymerization method. Compared to the previous system, the IBU loading capacity in the new POVI system was increased from 8.2 to $20.9 \%$, indicating that more IBU could be delivered into the tumor tissues when injecting the same amount of carriers.

First, we designed and synthesized a novel IBU-conjugated vinylbenzyl monomer, which was further polymerized to yield the IBU-containing block copolymers. Compared with the postconjugation method in which a drug was conjugated to the polymerized backbone, our method has an obvious advantage of simplicity. In addition, it avoids the low drug conjugation efficiency caused by the steric hindrance.

The synthesized POVI polymer could serve as a carrier that is effective in loading various types of chemotherapeutic drugs, including DOX, PTX, and DTX. We found that the monomer 
A

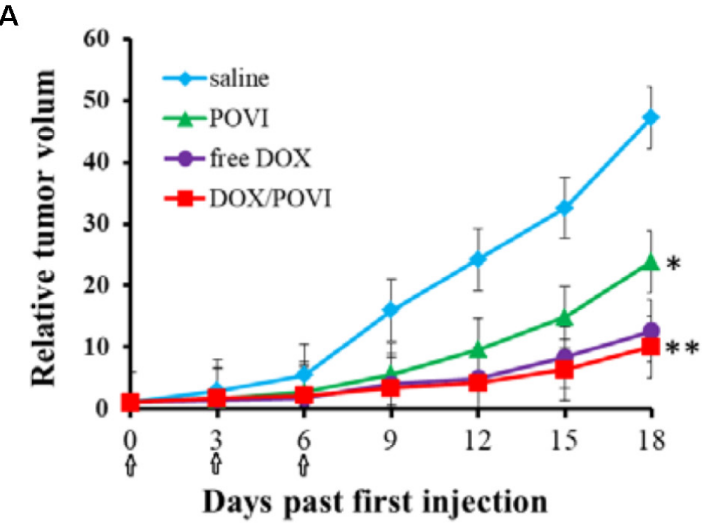

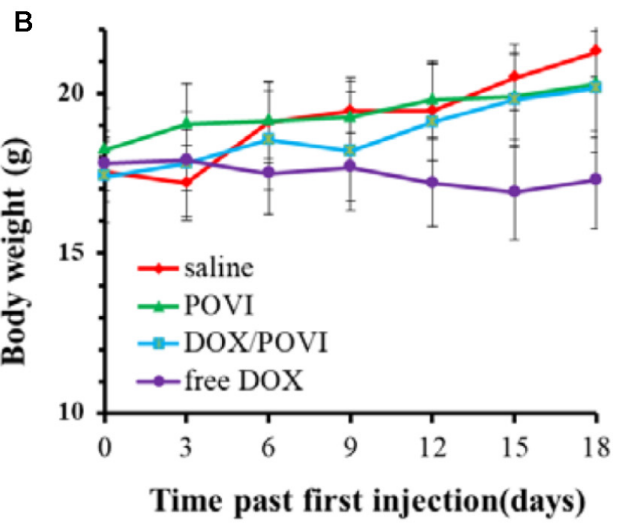

C

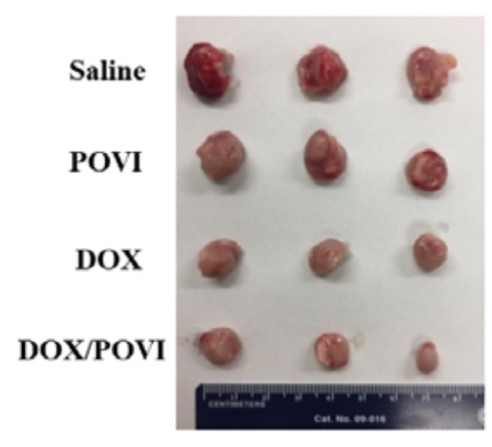

D

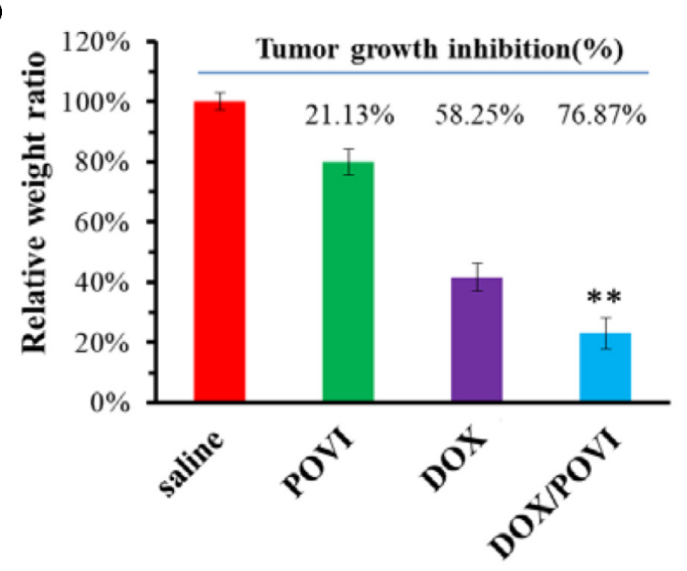

FIGURE 8| In vivo therapeutic efficacy of DOX/POVI micelles. BALB/c mice in 4T1.2 murine breast cancer model were injected with saline, POVI, free DOX, and DOX/POVI (5 mg DOX/kg body weight) on days 0,3 , and 6 , separately. ${ }^{*} P<0.05 ;{ }^{* *} P<0.01$ (vs saline). (A) Relative tumor volume curves of mice receiving different treatments. (B) Changes of body weight in mice were monitored. (C) Image of tumors stripped from mice on the day 18. (D) Tumor weights of mice were measured on day 21 and tumor growth inhibition (\%) was calculated. ${ }^{* *} P<0.05$ (vs free DOX).
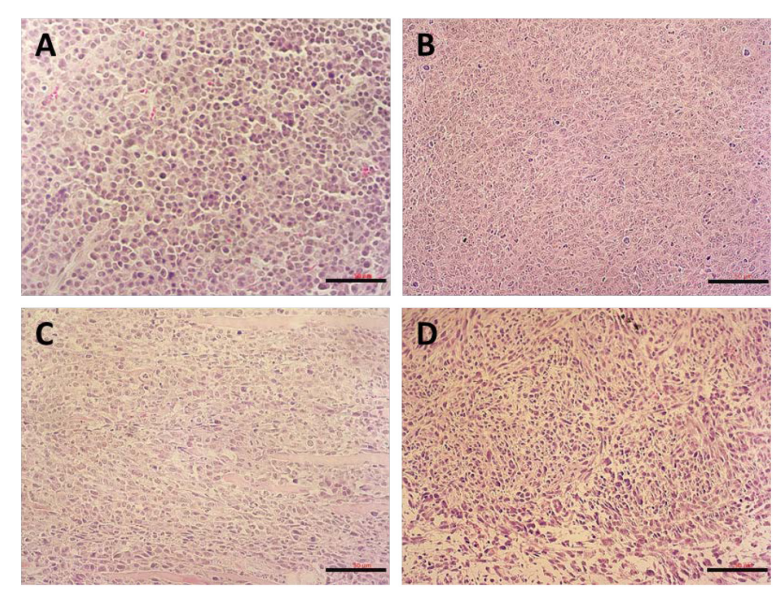

FIGURE 9 | H\&E stained tumor tissues from mice received different treatments: (A) saline, (B) POVI, (C) free DOX, and (D) DOX/POVI at a dosage of $5 \mathrm{mg} \mathrm{DOX} / \mathrm{kg}$. Three injections were made into BALB/c mice bearing $4 \mathrm{~T} 1.2$ tumor on days 0,3 , and 6 , separately. Tumor tissues were harvested on day 18. Scale bar: $50 \mu \mathrm{m}$. structure is critical for the loading capacity of the carrier. In the preliminary study, we also synthesized another IBUbased polymer using IBU-conjugated hydroxyethyl methacrylate monomer. However, the polymer obtained performed poorly in encapsulating other chemotherapeutic drugs (data not shown). When we introduced vinylbenzyl group into the polymers, the DLC was significantly improved, suggesting that, in addition to hydrophobic interaction, the $\pi-\pi$ stacking effect between carrier and the chemotherapeutic drug may also contribute to the overall DLC. Moreover, the structures of chemotherapeutic drugs also affected the loading capacity. The POVI carrier was more effective in encapsulating DOX compared to other chemotherapeutic drugs, which might be due to the stronger carrier/DOX interaction.

The DOX-loaded micelles showed small size with a diameter of $\sim 90 \mathrm{~nm}$ (Figure 2), which was optimal for a long blood circulation time and passive targeting to solid tumor sites through the enhanced permeability and retention (EPR) effect (Mitra et al., 2001; Meng et al., 2011; Kobayashi et al., 2014). DOX-loaded micelles showed slow and sustained drug release (Figure 5), which could prevent the premature release of 
encapsulated DOX before entering into tumors. The slow release of DOX from the micelles may be due to the strong interaction (e.g., hydrophobic interaction and $\pi-\pi$ stacking effect) between DOX and POVI carrier (Sun et al., 2016a).

In vitro cytotoxicity showed that DOX formulated in the carrier was less effective in inhibiting the proliferation of tumor cells compared to free DOX. The in vitro cytotoxicity comes from the overall outcome of intracellular uptake and drug release. The DOX/POVI micelles showed similar cellular uptake compared to free DOX (Figure 7); the lower in vitro cytotoxicity of DOX/POVI micelles might be attributed to the slow release of DOX from the micelles.

POVI carrier was not effective in inhibiting the proliferation of cultured tumor cells in vitro (Figure 6) at the concentrations that were used for DOX delivery in vitro. This may be due to the slow release of IBU during a short period of culture and its relatively low potency. However, in vivo data showed that the POVI carrier itself showed moderate antitumor activity (Figure 8). In our previous study, PEG-IBU conjugate could also inhibit tumor growth compared with control group (Zhao et al., 2016). So the antitumor activity of POVI carrier in vivo may come from the released IBU mediated by various kinds of enzymes in the tumor tissues over a relatively long period of time. Moreover, DOXloaded micelles showed a much more pronounced antitumor activity compared with free DOX, which is different from the in vitro cytotoxicity assay. The improved therapeutic efficacy of DOX/POVI micelles is likely attributed to the combination effect of the carrier with co-delivered DOX. The improved delivery of DOX by the POVI carrier may also play a role. More studies are warranted to further investigate the underlying mechanisms.

\section{REFERENCES}

Ahnen, D. J. (1998). Colon cancer prevention by NSAIDs: what is the mechanism of action? Eur. J. Surg. 164, 111-114. doi: 10.1080/11024159850191544

Andrews, J., Djakiew, D., Krygier, S., and Andrews, P. (2002). Superior effectiveness of ibuprofen compared with other NSAIDs for reducing the survival of human prostate cancer cells. Cancer Chemother. Pharmacol. 50, 277-284. doi: 10.1007/ s00280-002-0485-8

Bonelli, P., Tuccillo, F., Calemma, R., Pezzetti, F., Borrelli, A., Martinelli, R., et al. (2011). Changes in the gene expression profile of gastric cancer cells in response to ibuprofen: a gene pathway analysis. Pharmacogenomics J. 11, 412-428. doi: 10.1038/tpj.2010.55

Chen, Y., Xia, R., Huang, Y., Zhao, W., Li, J., Zhang, X., et al. (2016). An immunostimulatory dual-functional nanocarrier that improves cancer immunochemotherapy. Nat. Commun. 7:13443. doi: 10.1038/ncomms13443

Cho, K., Wang, X., Nie, S., and Shin, D. M. (2008). Therapeutic nanoparticles for drug delivery in cancer. Clin. Cancer Res. 14, 1310-1316. doi: 10.1158/10780432.CCR-07-1441

Craparo, E. F., Teresi, G., Licciardi, M., Bondí, M. L., and Cavallaro, G. (2013). Novel composed galactosylated nanodevices containing a ribavirin prodrug as hepatic cell-targeted carriers for HCV treatment. J. Biomed. Nanotechnol. 9, 1107-1122. doi: 10.1166/jbn.2013.1608

Davies, G., Martin, L.-A., Sacks, N., and Dowsett, M. (2002). Cyclooxygenase-2 (COX-2), aromatase and breast cancer: a possible role for COX-2 inhibitors in breast cancer chemoprevention. Ann. Oncol. 13, 669-678. doi: 10.1093/annonc/ mdf125

Dial, E. J., Doyen, J. R., and Lichtenberger, L. M. (2006). Phosphatidylcholineassociated nonsteroidal anti-inflammatory drugs (NSAIDs) inhibit DNA synthesis and the growth of colon cancer cells in vitro. Cancer Chemother. Pharmacol. 57, 295-300. doi: 10.1007/s00280-005-0048-x

\section{CONCLUSION}

We have developed a new IBU-based prodrug di-block polymer POVI via facile RAFT polymerization. POVI polymer could load various hydrophobic drugs including DOX, PTX, and DTX with high loading capacity. DOX/POVI micelles showed similar cytotoxicity and cellular uptake compared to free DOX. More importantly, DOX/POVI micelles were more effective in inhibiting the tumor growth than free DOX in vivo. Our results suggest that POVI polymer can be employed as a safe and effective dualfunctional carrier for co-delivery of other chemotherapeutic drugs.

\section{AUTHOR CONTRIBUTIONS}

SL, JS, and YH conceived and designed the study. JS and ZL contributed to chemical synthesis and micelle characterization. ZL, YL, JX, YC, LL, JL, and QL contributed to biological study. ZL, JS, YL, and JX analyzed the data. ZL, JS, and SL drafted the manuscript. All authors approved the final version of the manuscript.

\section{FUNDING}

This work was supported by NIH Grant Nos. RO1CA174305, RO1CA219399, and RO1CA223788.

Endo, H., Yano, M., Okumura, Y., and Kido, H. (2014). Ibuprofen enhances the anticancer activity of cisplatin in lung cancer cells by inhibiting the heat shock protein 70. Cell Death Dis. 5:e1027. doi: 10.1038/cddis.2013.550

Gately, S., and Kerbel, R. (2003). Therapeutic potential of selective cyclooxygenase2 inhibitors in the management of tumor angiogenesis. Prog. Exp. Tumor. Res. 37, 179-192. doi: 10.1159/000071373

Gaucher, G., Dufresne, M.-H., Sant, V. P., Kang, N., Maysinger, D., and Leroux, J.-C. (2005). Block copolymer micelles: preparation, characterization and application in drug delivery. J. Control Release 109, 169-188. doi: 10.1016/j. jconrel.2005.09.034

Hasegawa, U., Van Der Vlies, A. J., Wandrey, C., and Hubbell, J. A. (2013). Preparation of well-defined ibuprofen prodrug micelles by RAFT polymerization. Biomacromolecules 14, 3314-3320. doi: 10.1021/bm4009149

Janssen, A., Schiffmann, S., Birod, K., Maier, T. J., Wobst, I., Geisslinger, G., et al. (2008). p53 is important for the anti-proliferative effect of ibuprofen in colon carcinoma cells. Biochem. Biophys. Res. Commun. 365, 698-703. doi: 10.1016/j. bbrc.2007.11.051

Klaikherd, A., Nagamani, C., and Thayumanavan, S. (2009). Multi-stimuli sensitive amphiphilic block copolymer assemblies. J. Am. Chem. Soc. 131, 4830-4838. doi: $10.1021 /$ ja809475a

Kobayashi, H., Watanabe, R., and Choyke, P. L. (2014). Improving conventional enhanced permeability and retention (EPR) effects; what is the appropriate target? Theranostics 4, 81-89. doi: 10.7150/thno.7193

Koudelka, S., Knotigova, P. T., Masek, J., Prochazka, L., Lukac, R., Miller, A. D., et al. (2015). Liposomal delivery systems for anti-cancer analogues of vitamin E. J. Control Release 207, 59-69. doi: 10.1016/j.jconrel.2015.04.003

Lee, A., Di Mascolo, D., Francardi, M., Piccardi, F., Bandiera, T., and Decuzzi, P. (2016). Spherical polymeric nanoconstructs for combined chemotherapeutic and anti-inflammatory therapies. Nanomedicine 12, 2139-2147. doi: 10.1016/ j.nano.2016.05.012 
Lee, H., Lee, Y. S., Lee, K. D., and Park, S. Y. (2011). Development of disulfide core-crosslinked pluronic nanoparticles as an effective anticancer-drug-delivery system. Macromol. Biosci. 11, 1264-1271. doi: 10.1002/mabi.201100083

Lim, J. T., Piazza, G. A., Han, E. K.-H., Delohery, T. M., Li, H., Finn, T. S., et al. (1999). Sulindac derivatives inhibit growth and induce apoptosis in human prostate cancer cell lines. Biochem. Pharmacol. 58, 1097-1107. doi: 10.1016/ S0006-2952(99)00200-2

Meng, H., Xue, M., Xia, T., Ji, Z., Tarn, D. Y., Zink, J. I., et al. (2011). Use of size and a copolymer design feature to improve the biodistribution and the enhanced permeability and retention effect of doxorubicin-loaded mesoporous silica nanoparticles in a murine xenograft tumor model. ACS Nano 5, 4131-4144. doi: 10.1021/nn200809t

Miao, L., Guo, S., Lin, C. M., Liu, Q., and Huang, L. (2017). Nanoformulations for combination or cascade anticancer therapy. Adv. Drug Deliv. Rev. 115, 3-22. doi: 10.1016/j.addr.2017.06.003

Mitra, S., Gaur, U., Ghosh, P., and Maitra, A. (2001). Tumour targeted delivery of encapsulated dextran-doxorubicin conjugate using chitosan nanoparticles as carrier. J. Control Release 74, 317-323. doi: 10.1016/S0168-3659(01)00342-X

Rösler, A., Vandermeulen, G. W., and Klok, H.-A. (2012). Advanced drug delivery devices via self-assembly of amphiphilic block copolymers. Adv. Drug Deliv. Rev. 64, 270-279. doi: 10.1016/j.addr.2012.09.026

Senevirathne, S. A., Washington, K. E., Biewer, M. C., and Stefan, M. C. (2016). PEG based anti-cancer drug conjugated prodrug micelles for the delivery of anti-cancer agents. J. Mater. Chem. B 4, 360-370. doi: 10.1039/C5TB0 $2053 \mathrm{~K}$

Shen, Y., Jin, E., Zhang, B., Murphy, C. J., Sui, M., Zhao, J., et al. (2010). Prodrugs forming high drug loading multifunctional nanocapsules for intracellular cancer drug delivery. J. Am. Chem. Soc. 132, 4259-4265. doi: 10.1021/ ja909475m

Smalley, W. E., and DuBois, R. N. (1997). Colorectal cancer and nonsteroidal antiinflammatory drugs. Adv. Pharmacol. 39, 1-20. doi: 10.1016/S1054-3589(08) 60067-8

Sun, J., Chen, Y., Huang, Y., Zhao, W., Liu, Y., Venkataramanan, R., et al. (2017a). Programmable co-delivery of the immune checkpoint inhibitor NLG919 and chemotherapeutic doxorubicin via a redox-responsive immunostimulatory polymeric prodrug carrier. Acta Pharmacol. Sin. 38, 823-834. doi: 10.1038/aps. 2017.44

Sun, J., Liu, Y., Chen, Y., Zhao, W., Zhai, Q., Rathod, S., et al. (2017b). Doxorubicin delivered by a redox-responsive dasatinib-containing polymeric prodrug carrier for combination therapy. J. Control Release 258, 43-55. doi: 10.1016/j.jconrel.2017.05.006

Sun, J., Chen, Y., Li, K., Huang, Y., Fu, X., Zhang, X., et al. (2016a). A prodrug micellar carrier assembled from polymers with pendant farnesyl thiosalicylic acid moieties for improved delivery of paclitaxel. Acta Biomater. 43, 282-291. doi: 10.1016/j.actbio.2016.07.014

Sun, J., Luo, T., Sheng, R., Li, H., Wang, Z., and Cao, A. (2016b). Intracellular plasmid DNA delivery by self-assembled nanoparticles of amphiphilic PHML-b-PLLA-b-PHML copolymers and the endocytosis pathway analysis. J. Biomater. Appl. 31, 606-621.
Sun, J., Luo, T., Sheng, R., Li, H., and Cao, A. (2013a). Preparation of cationic 1-lysine conjugated poly (2-hydroxyethyl methacrylate) $s$ and their potential application as low cytotoxic efficient gene delivery vectors. J. Control Release 172:e108. doi: 10.1016/j.jconrel.2013.08.263

Sun, J., Luo, T., Sheng, R., Li, H., Chen, S., Hu, F., et al. (2013b). Preparation of functional water-soluble low-cytotoxic poly(methacrylate)s with pendant cationic l-lysines for efficient gene delivery. Macromol. Biosci. 13, 35-47. doi: 10.1002/mabi.201200304

Tucker, B. S., Getchell, S. G., Hill, M. R., and Sumerlin, B. S. (2015). Facile synthesis of drug-conjugated PHPMA core-crosslinked star polymers. Polym. Chem. 6, 4258-4263. doi: 10.1039/C5PY00497G

Ulrich, C. M., Bigler, J., and Potter, J. D. (2006). Non-steroidal anti-inflammatory drugs for cancer prevention: promise, perils and pharmacogenetics. Nat. Rev. Cancer 6, 130-140. doi: 10.1038/nrc1801

Wang, Y., Luo, Q., Gao, L., Gao, C., Du, H., Zha, G., et al. (2014). A facile strategy to prepare redox-responsive amphiphilic PEGylated prodrug with high drug loading content and low critical micelle concentration. Biomater. Sci. 2, 1367-1376. doi: 10.1039/C4BM00065J

Wang, Z., Luo, T., Sheng, R., Li, H., Sun, J., and Cao, A. (2015). Amphiphilic diblock terpolymer PMAgala-b-P (MAA-co-MAChol) s with attached galactose and cholesterol grafts and their intracellular $\mathrm{pH}$-responsive doxorubicin delivery. Biomacromolecules 17, 98-110. doi: 10.1021/acs.biomac.5b01227

Wang, Z., Sheng, R., Luo, T., Sun, J., and Cao, A. (2017). Synthesis and self-assembly of diblock glycopolypeptide analogues PMAgala-b-PBLG as multifunctional biomaterials for protein recognition, drug delivery and hepatoma cell targeting. Polym. Chem. 8, 472-484. doi: 10.1039/C6PY01526C

Wei, H., Zhuo, R.-X., and Zhang, X.-Z. (2013). Design and development of polymeric micelles with cleavable links for intracellular drug delivery. Prog. Polym. Sci. 38, 503-535. doi: 10.1016/j.progpolymsci.2012.07.002

Zhang, X., Huang, Y., Zhao, W., Chen, Y., Zhang, P., Li, J., et al. (2014). PEGfarnesyl thiosalicylic acid telodendrimer micelles as an improved formulation for targeted delivery of paclitaxel. Mol. Pharm. 11, 2807-2814. doi: 10.1021/ mp500181x

Zhao, M., Huang, Y., Chen, Y., Xu, J., Li, S., and Guo, X. (2016). PEGfmoc-ibuprofen conjugate as a dual functional nanomicellar carrier for paclitaxel. Bioconjug. Chem. 27, 2198-2205. doi: 10.1021/acs.bioconjchem. $6 \mathrm{~b} 00415$

Conflict of Interest Statement: The authors declare that the research was conducted in the absence of any commercial or financial relationships that could be construed as a potential conflict of interest.

Copyright (C) $2018 \mathrm{Li}$, Sun, Huang, Liu, Xu, Chen, Liang, Li, Liao, Li and Zhou. This is an open-access article distributed under the terms of the Creative Commons Attribution License (CC BY). The use, distribution or reproduction in other forums is permitted, provided the original author(s) and the copyright owner(s) are credited and that the original publication in this journal is cited, in accordance with accepted academic practice. No use, distribution or reproduction is permitted which does not comply with these terms. 